NISTIR 7732

\title{
Workflow and Electronic Health Records in Small Medical Practices
}

Mala Ramaiah

Information Technology Laboratory National Institute of Standards and Technology

Eswaran Subrahmanian Manufacturing Engineering Laboratory National Institute of Standards and Technology

Ram D. Sriram Manufacturing Engineering Laboratory National Institute of Standards and Technology

Bettijoyce B. Lide Information Technology Laboratory National Institute of Standards and Technology

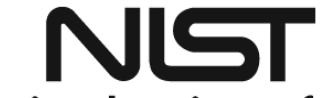

National Institute of Standards and Technology U.S. Department of Commerce 
NISTIR 7732

\section{Workflow and Electronic Health Records in Small Medical Practices}

Mala Ramaiah Information Technology Laboratory National Institute of Standards and Technology

Eswaran Subrahmanian Manufacturing Engineering Laboratory National Institute of Standards and Technology

Ram D. Sriram Manufacturing Engineering Laboratory National Institute of Standards and Technology

Bettijoyce B. Lide Information Technology Laboratory National Institute of Standards and Technology

November 2010

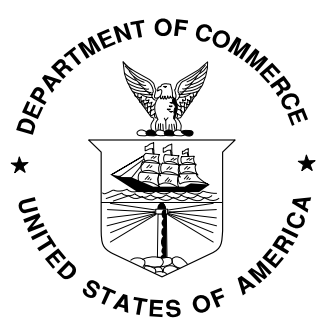

U.S. Department of Commerce Gary Locke, Secretary

National Institute of Standards and Technology Patrick D. Gallagher, Director 


\section{A b s t r a c t}

Objective: This study analyzes the workflow and implementation of Electronic Health Record (EHR) systems across different functions in small physician offices. We characterize the differences in the offices with different levels of computerization in terms of workflow, sources of time delay, and barriers to the support of the entire workflow through the use of EHR systems.

Design: The study was based on a combination of questionnaires, interviews, in situ observations and data collection efforts. The study was performed at 13 small practice physician offices in the Baltimore and New York areas. The offices included both primary care providers (4) and specialty providers (9). A total of 50 person-hours were spent with the staff to observe and capture the present work practice. This study was not intended to be a full-scale time-and-motion study with precise measurements, but to provide an overview of the potential sources of delays while performing office tasks.

Method: This study follows an interpretive model of case studies rather than a large sample statistical survey of practices. Workflow maps were created based on the aggregated data from the offices to identify time-consuming tasks. This information was used to compare and contrast the workflow maps for offices with and without EHR implementation. The workflow maps for specialist and primary care physicians were studied to understand the differences in workflow complexity that may be a factor in the differential adoption of EHR systems by the two types of offices.

Results: The results from the study show that specialty physicians are more favorable towards adopting EHR systems than primary care physicians. The results further indicate that while primary care physicians favored introducing EHR systems in their offices, the barriers to do so were greater than those encountered by specialists. This can be attributed to the complex workflows that exist in the primary care physician offices, leading to non-standardized workflow structures and practices. The results suggest that primary care physicians would benefit more from EHR systems if they could interact with external entities through computer-based transactions. Presently, non-standard ways of interaction with external entities and inadequate computerization are the primary barriers to moving away from paper-based systems. Without being able to move away from a paper-based system, an EHR system becomes an extra burden and results in low rates of adoption.

Conclusions: Significant variations were observed in workflow characteristics between primary care and specialty physicians that could be attributed to the variety and multiplicity of external interactions. It is easier to adopt an off-the shelf system if the variety and number of interactions are constrained. The manual nature of the tasks in non-EHR-based offices results in considerable waste of time and increase in cost. Any move to an EHR system should hence include a strategy for communicating with the outside world. 


\section{Introduction}

Healthcare is a continuing and evolving industry facing major challenges: increasing costs, unacceptably high error rates, and dissatisfied patients and providers [1]. Spending on healthcare in the United States continues to grow at the rate of over 7\% per year, expecting to reach $\$ 2.8$ trillion by 2011, around $17 \%$ of the Gross Domestic Product. Information technology is becoming essential in managing the healthcare enterprise [2]. Many hospital systems have adopted the use of the Electronic Health Record (EHR) systems, but there is very slow adoption in the case of individual or small group offices. A 2007 National Ambulatory Medical Care Survey reports that in over 161,200 medical practices in the U.S., there were 311,200 physicians practicing in office-based environments [3]. The report also identifies that $69.2 \%$ of practices consisted of individual practitioners. About $19.5 \%$ of the practices contained three or more physicians. Specialty practices accounted for about $8.4 \%$ of the total. About $19 \%$ of the physicians used EHR systems and 9.2\% used computerized prescription order entry systems [3].

Various studies conducted across a variety of healthcare settings have claimed that the introduction of EHR systems can lead to an improvement in quality and has improved operational efficiency and workflow [2]. In spite of these claims, the overall adoption rates of EHR systems have been reported to be as low as 17\% [4]. A report on the "Adoption Gap" claims that less than $11.3 \%$ of the small physician offices have fully implemented EHR systems [5]. Given that $88 \%$ of all medical offices are small practices, the report also identifies barriers to adoption. One of the main barriers identified is the nature and variety of workflow in the small physician offices, leading to resistance to incorporating EHR systems that offer little flexibility. The report also found that successful adoption requires close attention to office workflow, the way tasks are organized and resources used to achieve outcomes along with appropriate change management, planning and process re-engineering [5]. Finally, the report urges a more detailed case study of workflows. In the spirit of the questions raised by this previous study with respect to use of EHR systems, our paper complementarily summarizes case studies of workflow conducted in 13 physician offices (4 primary care and 9 specialist offices) over a three-month period.

Studies in the area of EHR system use vary from those that estimate huge costs savings [2] to those that disagree with the cost savings estimates and discuss problems with adoption [6,7]. Some studies point to poor current incentive structures and argue for new incentives to physicians so as to overcome the problems associated with inadequate capital, numerous vendors, lack of interoperability, misaligned incentive structures, fears of privacy, and perception of economic benefits by the adoption of EHR systems [8, 9, 10, 11, 12]. While these studies point to the diversity of barriers to adoption, the focus of this paper is to develop a deeper insight into understanding the workflow, the operational mode in completing a task, the effect of context (primary care vs. specialty offices), and the interactions with external entities that are part of the workflow. Based on our observations, this paper identifies the nature of the workflow and the information management problems in individual or small group practices. These observations are intended to aid in the re-engineering of the office organizations that will affect the adoption of EHR systems. 
The paper is organized as follows: in section 2 we elaborate on the study focus and design including the research questions addressed. Section 3 describes and provides an analysis of the workflow for a typical primary care office and three specialty offices and draws generalizations from these specific cases to the rest of the use cases. In section 4 we summarize the results in terms of the research questions that were framed in the study focus section. Section 5 finally presents some general conclusions.

\section{Study Focus and Design}

Several approaches have been used to studying work practices and productivity of physicians' offices. Most of the studies reported in the literature use standard survey methods to generate statistically significant conclusions based on adoption, costs, barriers and other aspects of the implementation of EHR systems [12, 13, 14, 15, 16, 17]. Some other studies are qualitative in nature. In the study presented in this paper, we have taken an interpretive case study approach so as to get an in-depth understanding of the workflows, the current role of EHR technology, and the external factors or interactions that affect workflow automation. Interpretive case studies have been in extensive use in social sciences and management literature. Yin has developed a set of guidelines on the role of interpretive case studies in the social sciences [18]. Case studies are used not to create a statistically based hypothesis-testing exploration but as a means to answer specific research questions through a combination of methods such as questionnaires, interviews and observations of the object of study. Generally, the goal of interpretive case studies is to answer an identified set of questions in the context of previous studies conducted by other researchers. Our intent in using the interpretive approach is to additionally provide a deeper understanding of similar research questions.

To set the stage for reporting on the data collected and the methods used in the study, we clarify the definition of workflow used in this paper. We choose the definition of 'workflow' as defining the interaction patterns among a practice's staff as they fulfill tasks and produce outcomes using available resources [5]. Using this definition, we identify the tasks conducted by the entire physician's office from the arrival of the patient to his or her departure. We also identify the tasks that are the sources of delay, either due to the nature of the task or to external events, such as additional unanticipated transactions required to complete the task. This approach to studying small physician offices allows us to understand the nature and variety of workflow in different types of offices, the time delays inherent in the processes, and the resources available to complete tasks. We also identify those tasks where automation led or can lead to specific benefits for small practices.

Given the above perspective of our study, the research questions asked are:

1. What are the variations among specialist and primary care offices in terms of workflow?

2. What are the sources of delay or most time-consuming tasks that are part of the workflow?

3. What are the reasons for the differences in the extent of computerization in specialist and primary care offices? 
We address the above research questions by combining the method of using questionnaires with in situ observations and interviews. The design of the questionnaire was meant to facilitate elicitation of the workflow in as clear a fashion as possible to account for the interaction patterns of the staff and the physician in the offices. We used the data and observations to create workflow maps for each office with the mode (e. g., paper, telephone, computer) in achieving the tasks in the workflow. We also identified the tasks that used some form of computerization. In our interviews, we verified the workflow constructed from the questionnaire and probed the causes of delays in the time-consuming tasks through staff interview.

The workflow diagrams were visualized using Universal Modeling Language (UML) activity diagrams to provide a common scheme for representing them across offices [19]. The names of the tasks, if used differently by the different offices, were normalized to mean the same across the cases. Once all of the data were organized and analyzed, we used the interview notes to elaborate on the answers to specific questions. Subsequently, we placed the results of our study in the context of other studies reported in the literature.

The interviews and observations were conducted in situ to validate the tasks and workflow patterns in the physician offices. This study was done at 14 small practice physicians' offices in the Baltimore and New York areas. The set of physicians' offices included both primary care (4) and specialty providers (9). A total of 77 additional person-hours were spent with the staff to interview, observe and capture the present work practices of these offices. In the offices where we conducted detailed studies, the workflow was generalized and the time taken for tasks noted and averaged for about 10 patients. The study is not intended to be a full-scale time-and-motion study with precise measurements, but is rather intended to provide an overview of the potential sources of delays and the proportion of time taken by the different activities in the workflow.

\section{Workflow Analysis and Observations}

The subsequent sections present the background data of the study and workflow analysis in the physician offices studied. Here, we also try to compare among the physician offices studied and variations among the offices in terms of workflow.

\subsection{Background Data}

For a uniform presentation format, the first step in the study was to identify the tasks performed in the offices and give a consistent term for each task across the practices. This is essential because the questionnaires were designed for maximum freedom to the respondents and did not constrain the answers to a particular terminology. The standardized terms for various tasks were then verified during questionnaire analysis to have the same meaning as the ones provided by all of the offices under study. Table 1 below gives a composition of people by function and facility in the physicians' offices. On an average, there are two staff persons per physician in primary care offices (cols.2-5) and about three persons per physician in specialty care offices (cols. 6-14), as they tend to perform additional services in-house. In some cases, the front desk tasks, e.g., registration, billing and information organization, are performed by the same person, and in other cases there are specialized staff people for billing and recordkeeping. These variations seem to arise from the manner in which a physician's office evolved. Table 2 provides the tasks and task handling modes across different offices. In our study of 13 offices, four offices had implemented EHR systems; three had no computerized approach whatsoever; while the other six offices had 
some form of minimal computer usage (partial implementation). It must be noted, however, that the four offices with an EHR implementation were in their initial stages (less than 6 months of implementation). As can be seen in Table 2, only one of the family-practice/primary care physicians had some form of computerization. In offices that provide services for a specialty, the range of computerization of tasks varied. Some offices were in the process of implementation by computerizing a partial set of tasks. In general, the use of paper and telephone was pervasive across the offices when dealing with external entities such as insurance companies, referrals to other doctors, and laboratories.

Table 1: Physician Office Compositions (attached at the end)

Table 2: Small Office Physician Tasks and Mode of Work (attached at the end)

\subsection{Workflow Analysis}

In this section we take three specific offices, a primary care office, multi-specialty office and a specialty office, and describe the work pattern observed. We present the workflow using UML activity diagrams. Among the three offices chosen, two of them represent the two extremes in terms of computerization and the other shows partial computerization. These specific cases serve as exemplars of the type of workflow that exists. It must be noted that while other offices are not described in detail here, they have certain characteristics in common, with slightly different organizational structures, based on the combination of services offered within the office and those that are external, leading to variations in the workflow, especially when contrasting primary care physician and specialist offices. This does not imply that there are no differences among the offices within each category, but rather that the differences across the categories are much more significant. After presenting the individual cases we explore the differences. In addition, we also discuss the variances observed in the workflow among offices at different locations, implying that the offices adapt to the available resources and the practices of the external services. In the subsequent section, we present the case study in detail with respect to the tasks performed and the sources of delay.

\subsubsection{Work Practices and Workflow in a Primary Care Provider Office}

The workflow in the primary care offices is generally difficult to capture in its entirety with respect to the time taken for each specific task related to each patient. This particular office (Primary Care - 1 in Table 2) embodies one end of the spectrum where there is no computational support for any task. The office had two primary care physicians with different weekly visiting schedules and was staffed with two full-time front desk clerks and one nurse. There was no computer facility in the office and hence everything was done manually. The nurse also manages the front desk activities when needed. The staff works amidst heaps of forms, files, and paper. There appears to be almost every chance for important reports to get lost among the paperwork (this happened once during the observation study; there was a missing lab report that had earlier been received; hence, the clerk had to collect the report from the laboratory again, and spent considerable time on the telephone). 


\subsubsection{Description of Tasks}

The descriptions of the tasks listed below are based on the roles played by participants. Variation among primary care offices with respect to these roles was minimal or non-existent.

\section{Front Desk Clerk}

1. Scheduling appointments: Scheduling visits for first-time patients is performed over the telephone for the first available mutually convenient time; basic demographic information is also collected on this call. Scheduling of follow-up appointments is done at the end of the visit, based on the doctor's verbal instruction to the patient. The clerk checks an appropriate date in the paper-based planner and comes to a mutual agreement with the patient. The appointment date and time are entered into the planner and a card with the appointment information is given to the patient. The patient is telephoned three days before the appointment for confirmation or rescheduling. In the case of cancellation of appointments by the physician, the clerk telephones the patients and reschedules them.

2. New patient registration: The process of new patient registration is cumbersome and requires the collection of an extensive amount of additional data. The patient furnishes insurance details, demographic details, and previous health records/history. The insurance card furnished by the patient is taken, a copy made, filed, and a detailed verification whether the insurance is active is done. The patient fills in the demographic and health history details on a paper demographic sheet. The history is limited to the information provided by the patient until the records are received from the previous primary care physician (PCP)'s office after an authorization by the patient to release the records. The clerk files this sheet in the patient's chart and makes a copy to be sent to the insurance company for the claim associated with that day's visit.

3. Repeat patient registration: A repeat patient signs the sign-in sheet and then waits for the next step. The clerk retrieves the patient's chart, makes copies of the demographic sheets, insurance details and billing encounters, and files them to the chart. The clerk deposits the newly constituted chart in the nurse's station for subsequent processing of the patient.

4. Insurance verification: Insurance verifications by telephone inevitably lead to delays associated with routing the call to the proper person. The clerk has to make inquiries not just about the validity of the insurance and the scope of coverage, but also that the physician is certified to be paid. In some cases, the insurance company that has a prior relationship with the physicians' office sends the list of patients covered by the company for each physician in the office. This allows for an easier verification of insurance information. However, missing patient's or doctor's name in the list due to outdated lists lead to various delays associated with contacting the insurance company with long holds over the telephone.

5. Referrals: The clerk prepares the referral to a laboratory for tests or to another physician requested by the examining physician. In the case of referral to a laboratory, the patient waits until the referral request, with details of the required test and insurance, is generated. For laboratories that do not allow for walk-in patients, an appointment for the 
test is also scheduled. In the case of referral for a consultation with another physician, a hospital, or specialized tests such as a CAT (Computer-Aided Tomography) scan or a PET (Positron-Emission Tomography) scan, the patient is contacted with the details later. In this case, the clerk clears this referral later in the day by contacting the appropriate physician, laboratory or hospital for scheduling an appointment. Then the clerk either faxes the patient information to the respective entity or mails the information. The patient is informed by telephone about the appointment and the referral sheet is mailed to the patient.

6. Organization and collation of received reports and information: On a daily basis, the office receives numerous reports and pieces of information by fax and mail from laboratories, insurance companies, and patients, as well as other practice-related information. Without any prioritization, the physicians receive these reports for review. The physician checks these at his convenience. Upon review, he initials the reports and instructs the clerk to generate one of several actions, such as requesting the patients' charts for an immediate review, scheduling an immediate appointment with the patient, requesting hospitalization for emergencies or writing referrals based on the abnormality or other conditions observed in a report. Most of the time, the physician reviews the reports several days after receipt.

7. Prescription-related activities: Physicians hand-write most prescriptions and give them to the patient. Some prescriptions are faxed to the pharmacy at the request of the patient. While a relatively simple task, there are often differences in dosage or medications covered by insurance companies, and the prescription requires clarifications.

8. Organizing the charts: The clerk reviews a patient's chart after each consultation, to identify any action items that were not completed. If no action items are outstanding, the chart is filed. Else, attempts to complete the actions are taken. Records of the encounters are filed; insurance details are copied and put on the chart to be sent for billing. This task is usually interleaved with regular work or performed during lunchtime.

9. Mailing letters: The referral orders or any other informational letters such as upcoming appointments are mailed to the patient as well as to the referred organizations.

10. Copying, faxing, and attending telephone calls: This is a major front desk activity, with tasks interleaved throughout the workday.

11. Issuing certificates: As per the physician's orders, the front desk clerk issues certificates such as back-to-school/work or sickness certificates.

Nurse

1. Collecting brief illness history and recording vital signs: The patient proceeds to the nurse after the initial registration process. The nurse usually solicits a brief history of illness, records vital signs, and enters them in the chart. The nurse verbally informs the physician of any important findings. 
2. Collecting co-pay: The nurse also collects the co-payments.

3. Front desk activities: The nurse assists with front desk activities as time permits.

\section{Physician}

1. Routine history and examination: The physician carries out the routine examination of the patient and advises the patient accordingly.

2. Entering progress notes: The physician enters progress notes of the consultation after examining the patient and updates the medication record as required.

3. Entering billing details: The physician records the billing details in the chart after the patient's visit. This information is used by the front desk to be sent to the billing agency.

4. Communication with the fellow consultants and hospitals: The physician's activity also involves communicating with fellow consultants and hospitals to discuss the patient's condition and to issue orders for hospitalization when needed.

5. Reviewing faxes and issuing follow-up actions: The physician reviews the faxes and mail and issues appropriate orders.

Figure 1 depicts the workflow diagram of this physician's office using an UML activity diagram. One important caveat is that some of the tasks are interleaved across patients, such as the collection/collation of records, making precise measurements of time taken for each task and for each individual patient difficult. Data on the time taken for each task were not collected as some of the tasks were time-sliced. For example, while waiting for the insurance company to answer, the front desk clerk may process another application. Time-slicing takes place throughout the day, especially with the organization and clearing of documents for distribution within and outside the office.

We have included a second view of workflow. The diagram in Figure 2 illustrates the interaction and information flow, identifying the time-consuming tasks and some of the external and internal interactions. 


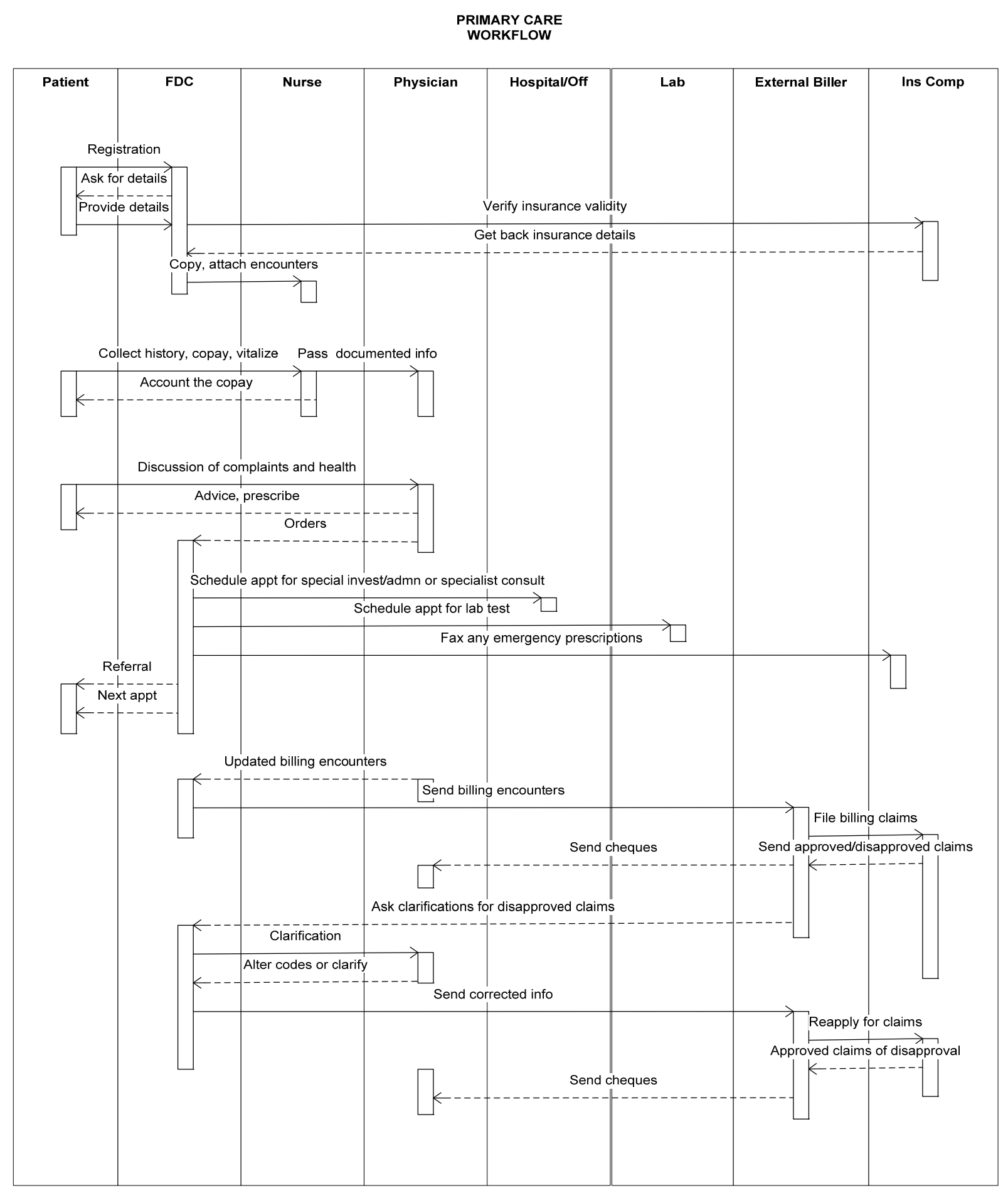

Figure 1: UML Based Activity Diagram for Primary Care Office 


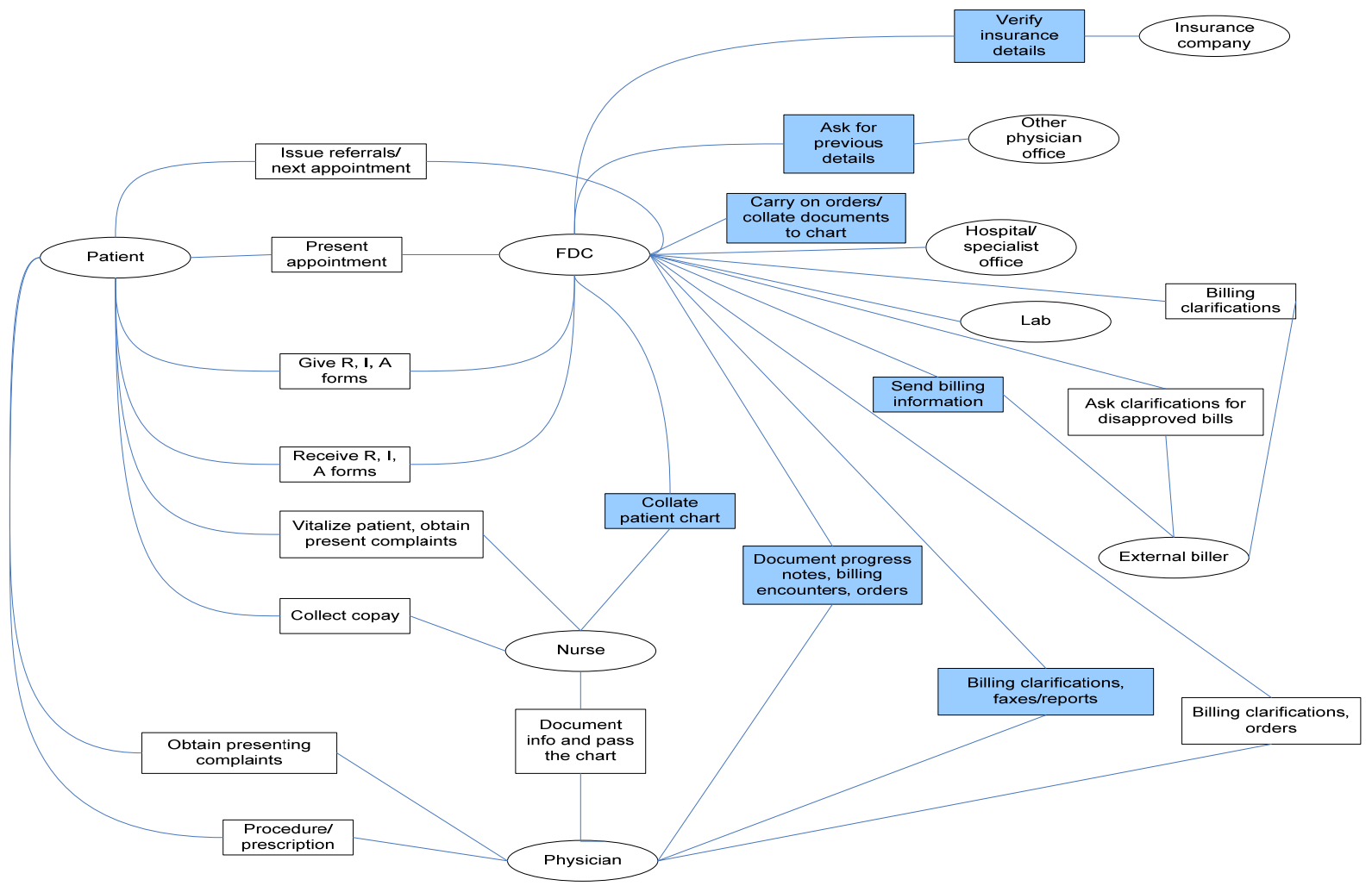

Figure 2: Interaction Diagram Showing the Primary Sources of Delay

\subsubsection{Sources of Delay in the Workflow Process by Task}

The following are the primary sources of delay in the workflow process of the office studied:

1. Scheduling: The office maintains two paper-based planners, one for each physician. There is a potential chance to enter the appointment in the wrong physician's planner due to oversight. The paper-based planners, the specific physician-based entries and the searching and rescheduling of appointments lead to a time-consuming scheduling process. For example, if a patient forgets the appointment date the clerk has to meticulously search through the planner.

2. New Patient/Repeat Registration: In this step, the physician is limited by the information he/she receives from the patient and its validity until he/she receives the entire record, leading to possible incorrect diagnoses and quality-of-service problems. Further, the process of collecting information from previous physicians who have examined the patient is time consuming, often requiring follow-up telephone calls. 
For a repeat patient, making copies of a prior chart for update maintains the continuity in the patient's record; however, this wastes both time and paper. Maintaining multiple copies of the record over time could lead to misfiling or loss of documents.

For the registration task, the primary problem is again organization and management of information. All the information delivered to the doctors is delivered on paper and hence has to be organized and collated; new information from the patient has to be gathered for registration. The front desk clerk spends a lot of time organizing information on paper, leading to a propensity for errors and incomplete information. During our observation, there was an instance where the demographic details were not updated; a letter sent to the patient was not received and the prescribed test was not performed.

3. Insurance Verification: Multiple insurance companies and the variability in coverage and participation of the physicians in a particular insurance company program increase the complexity of insurance verification. The front desk clerk has to maintain and search multiple insurance lists that may or may not be up-to-date at the time of registration. This leads to a very time-consuming and error-prone process.

Additional time is spent by the physician for additional approval for tests and for further consultations with doctors working for the insurance companies, even after the examination of the patient. This interaction with insurance companies is a major bottleneck, creating confusion and conflicts in small offices.

4. Referrals: The referral process is cumbersome, time consuming and error prone because a number of factors play a role in determining the workflow for a particular case. The clerk not only has to identify the laboratory or physician to be contacted, but also whether a given insurance company certifies the laboratory or physician as an authorized supplier of the service.

5. Organizing and collation of received reports: Organizing and prioritizing the reports and other information is a very time-consuming task requiring attention on the part of the front desk clerk and the physician. While a front desk clerk may be able to organize and prioritize the information to some extent, he/she is generally not competent enough to make a judgment on the prioritization of patient appointments. Once the physician has reviewed the information, a number of possible workflow items needs to be generated depending on the information received on a given patient. Delays in reviewing the reports and information could lead to errors and delays in care.

6. Progress notes: In most offices, the documentation of the physician's interaction with a patient is not immediately entered into the charts. It is normally done either after all the consultations for the day or may take 2 to 3 days. The delay in documentation of the consultation, in spite of it being the most salient feature in the chart, could lead to information loss and mistakes.

7. Prescription related activities: Pharmacists often need clarification from the physician. This task results in considerable interruptions to the physicians. 
8. Other unscheduled tasks (mailing, organizing patient charts, copying, faxing, issuing of certificates and attending to telephone calls): This class of tasks is inherently scheduled in an ad hoc manner. They are done as and when required or when time permits. Clearly, the tasks of faxing, copying and issuance of certificates require manual work and in a paper-based office require moving, writing, and filling forms and performing tasks that take time. Not all of them can be completely re-engineered, especially managing telephone calls.

\subsubsection{Observations across Primary Care Offices}

We studied three additional primary/family practice physicians in a manner similar to the exemplar case presented. We observed heterogeneity among primary care offices as well as in the functioning of individual offices. The following are the observations common to all these offices:

1. Each office functions in its unique way. Therefore, the workflow is not uniform even though tasks and some processes match. Further, the workflow is different in different regions, depending on the practices and protocols adopted by the laboratories and insurance companies of that region. In contrast to the observed offices in Maryland, where patients are referred to a laboratory for blood sample collection, in New York the physician's office collects the samples and the clinical laboratory usually picks them up by evening and sends the reports the next day. In addition, the insurance companies follow different protocols ranging from telephone calls for the verification of patient information to some that use verification machines (similar to a credit card) for getting approval using the patient's insurance card.

2. In all primary care offices, the workflow pattern is different for different patients depending on their condition. The patient with any physical condition or symptom first comes to the PCP (primary care physician). Each patient may have a different treatment protocol. Hence, there are different workflow patterns and different kinds of referrals. The workflow pattern is different for regular patients, for patients referred from nursing homes, and for patients who come through Medicare. The tasks of registering or referring to a hospital vary due to the differences in the patient's requirements.

3. In the primary care/family practice offices studied, there was no or minimal amount of computerization. In some cases, the billing process was outsourced to a billing agent for creating comprehensive reports and follow-on monitoring. While this does reduce some paper work it still requires manual processing (Table 2).

The above observations confirm the results from the previous studies on the low adoption of EHR systems in primary care physician offices [5]. In our interviews with various physician facilities, the primary reasons for not adopting EHR systems were the lack of IT experience and the uncertainty of the time and costs involved. However, the physicians expressed a belief that they would be able to improve their operations with IT. The general feeling among these offices is that using EHR systems would not result in reduced physician interaction time. The 
observation is in agreement with the results of time-and-motion studies of physicians in a primary care office, which show that there is very little decline in the time spent by the physician with a patient with the EHR implementation [14]. Hence, from a primary care point of view, unless the incentive structure for reimbursements is changed and interfaces to external entities are made seamless, it is unlikely that EHR systems will be widely adopted.

\subsection{Work Practices and Workflow in Specialty Care Offices}

Nine specialty care offices were studied. They included specialty practices in Neurology, Obstetrics and Gynecology, Gastroenterology, Cardiology, Hematology/Oncology, Ophthalmology, Nephrology, ENT (ear, nose, and throat) and a Multi-specialty office. In this section we present the workflow and task structure in three specialty offices that vary in their level of computerization: a multi-specialty office with relatively minimal computerization, a hematology/oncology office with partial computerization, and an ENT office with the most advanced computerization.

\subsubsection{Workflow Analysis: Multi-specialty Office}

The multi-specialty office observed is a unit of the Medicare Waiver Program and has been in existence for the past 5 years. It is attached to a local hospital. There is a separate room and staff where records are maintained. The unit has a billing system of its own. This multi-specialty office also has its own sample collection, X-ray, and pharmacy centers. FoxPro is the software used to schedule appointments. The software has several features that are not regularly used; only features such as tracing previous appointments and monitoring copay from previous visits are used. In the future, this office is expected to be split into individual specialty offices, a separate privatized billing office and in-house laboratories.

\section{Front desk clerk}

The front desk clerk spends most of the time on scheduling appointments using FoxPro. The charts are sent from the record section; as the patient arrives, the chart is put in the rack for the appropriate physician. The front desk clerk is also involved with the new patient registration, using the same software. However, paper printouts are put into the charts as well. The front desk clerk is also responsible for collecting copay and making inquiries about patient demographics.

Record section

The record section has two people to take care of the entire process of record maintenance. They pull out the charts for all the physicians for the coming day, add required encounters and pass the charts on to the front desk at the appropriate time. They also are equipped with a computer with the FoxPro database application, where they sometimes make inquiries into the details of previous visits by a patient.

Nurse 
Each physician has his or her own nurse. The nurse is responsible for pulling up the laboratory results from the hospital's website, taking printouts and filing them, collecting faxes and giving them to the physician, scheduling appointments for referrals and special investigations, issuing forms and certificates, sometimes faxing the prescription, and also obtaining vital statistics of the patient and helping the physician with some procedures.

\section{Physician}

Most of the physicians in the office do not use computers for their progress note entries nor for prescriptions.

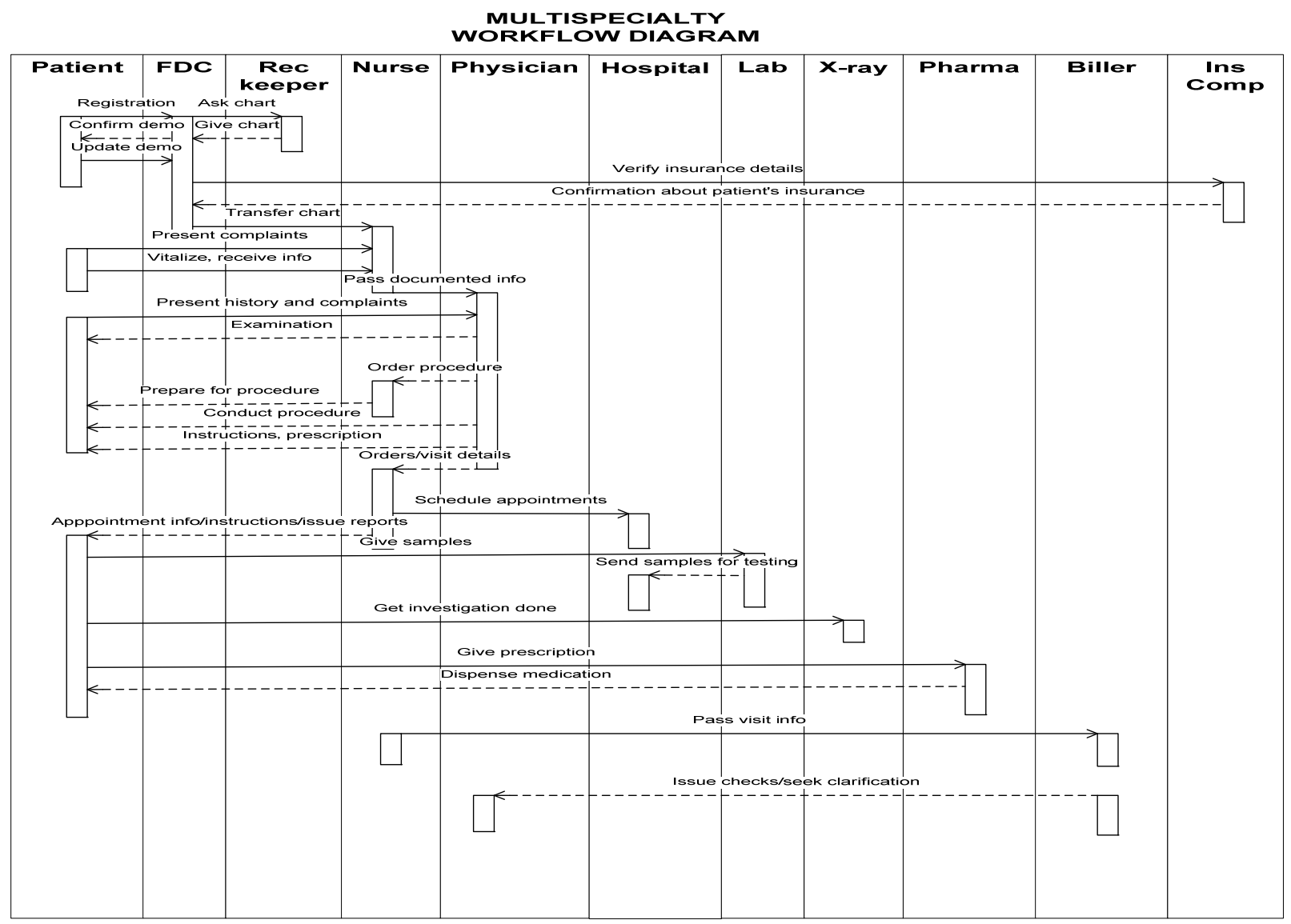

Figure 3. UML Activity Diagram for Multi-Specialty Office

Figure 3 is UML activity diagram for the tasks of this office. The interesting aspect of this office is that it is partially computerized with respect to scheduling of appointments, billing and interactions with the laboratory. The health records part of the office is not computerized and is still on paper. At the time of this case study, it was noticed that there were plans to split the 
currently integrated office into privatized functional pieces. Further computerization will depend on the strategy adopted by the individual physicians. However, as the office exists today, it is well integrated with respect to laboratory information and has its own X-ray facilities, which makes information collection easier. Scheduling was the office's first effort at computerization as it allows for coordination across specialists and internal laboratory facilities. This has resulted in considerable time savings. The office has islands of computerization, including a billing system provided by the hospital. This fragmentation poses a problem as data from each of the computer systems still need to be collated in one place and this is done with paper.

\subsubsection{Workflow Analysis: ENT Office}

The ENT Office is a single practice office with some procedures, such as audiometry, ultrasound assessment, FNAC, small surgical procedures performed in the office. The staff includes five members: 2 front desk clerks, 1 nurse, 1 audiologist, and 1 billing person. This specialty office was the most computerized/automated office among those studied. The office uses a primitive EHR system for scheduling, sharing documents, and billing. The progress and procedure notes are transcribed, then scanned and put in the EHR system.

\section{Front desk clerk}

The work at the front desk is carried out by a staff proficient in using the EHR system. The chart of the patient is a thin folder containing the printout of the previous visit notes and the previous laboratory reports for the physician's reference during the examination. This is because the physician does not have a computer in the examination rooms but has one in his/her private office. The chart is not stacked up with all the reports but rather contains only the paperwork associated with the previous encounter. The front desk staff creates this chart once the patient arrives for consultation. The patient registration and sign-in verification is done directly in the EHR system. The other front desk activities such as investigation forms, issuing certificates, scheduling appointments are done through the computerized system, but the telephone is used for referral and scheduling appointments to other centers.

Nurse

The nurse is in charge taking the patient's vital signs and carrying on investigations, such as audiometry for the patient.

\section{Billing agent}

The billing agent present in the office works solely for this physician's practice. He/she gets immediate information about patient visits in the office. He/she also gets the information about the hospitalized patients and sends bills to the insurance companies that accept e-bills. Otherwise, he/she sends the billing information by mail.

\section{Physician}


The physician examines the patients and dictates the notes over a Dictaphone for transcription. $\mathrm{He} / \mathrm{she}$ completes this during the consultation. Therefore, there is no additional work after consultation. Prescriptions are paper-based.

The current EHR system used, while primitive in some respects, is the repository of all patient information including the transcribed doctor's notes. The staff finds it easy to work with the current EHR system, but is not happy with the performance of the system as it is comparatively slow with respect to working on paper and hinders work sometimes. The consultation notes are entered into the EHR system by using transcription services. We got the impression that the staff felt that the EHR system use results in better work management and easier retrieval of patient records. The staff also felt that the use of the EHR system has improved performance in terms of time taken for various tasks, in contrast to the previous workflow model. Even though this office had the highest level of computerization of all the offices we visited, it still had problems in dealing with laboratory referrals and other external interactions that take place on paper. The office scans all of this information. This may reduce search time in the future, but does not help in time reduction for organizing and collating information from external sources.

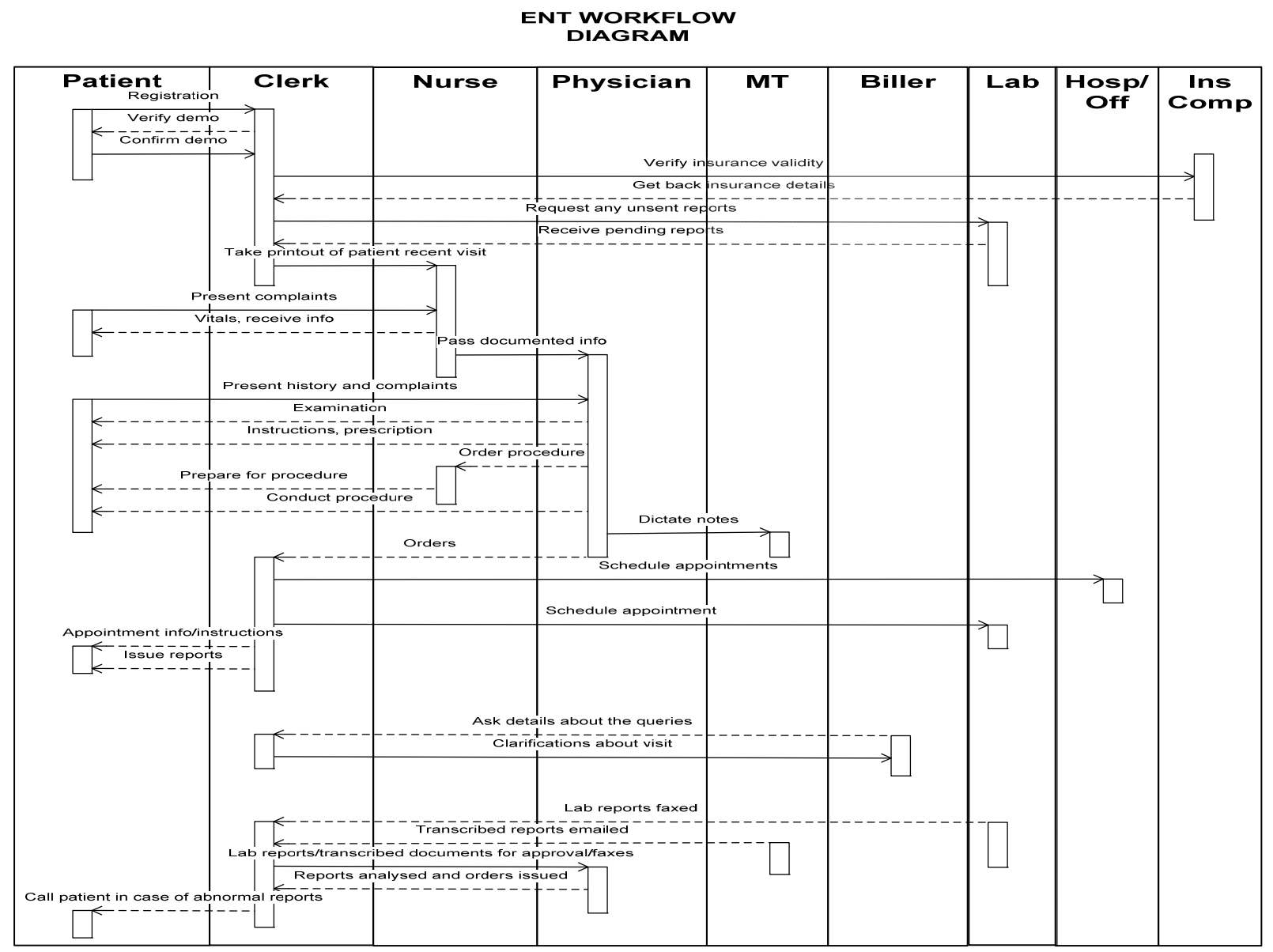

Figure 5. UML Activity Diagram for ENT Specialty Office 
Figure 5 shows the UML activity diagram for the ENT office. The level of computerization is high, but the primitiveness of the system being used reduces the efficiency that would have been achieved with a better selection of the EHR system.

\subsection{Observations from Specialty Care Offices}

Nine specialty care offices were studied, including the ones described above. A significant aspect of a specialty care office is that it deals with a specific human system. Hence, the range of workflow is often limited. The specialties being narrowly focused, in spite of the multiplicity of sources of patient input (PCP, internists, etc.), they have more limited external interactions and are less varied in their transactions. All the specialty care offices we studied had scheduling software with varying degrees of automation of tasks, as shown in Table 2.

Table 2 illustrates the level of computerization in the offices with the ENT office being the most computerized. Nevertheless, even in the ENT office, all tasks involving external interactions such as insurance verification, prescription, external referrals and laboratory test requests are still paper-based. The only exception is the multi-specialty office that has a computer-based interface with the laboratory, because the office is connected to a hospital that provides the laboratory service. It is also interesting to observe that in all of the specialty offices, the appointment scheduling and registration tasks were computerized. The other task that was mostly computerized is the billing task, except for the two offices (Neurology and Gastroenterology) that used external billing services.

A significant difference that we found between the specialty offices and the primary care offices is that most of the specialty offices tend to have diagnostic testing services, or other outpatient therapy services, in-house. For example, the oncology specialty has facilities for outpatient chemotherapy service, in-house and the ophthalmology specialist performs ophthalmic procedures on site. In addition, the multi-specialty office services include a sample collection center for laboratory samples, and X-ray and pharmacy centers. While each specialty is different, they seem to integrate some of their services in-house for the convenience of patients as well as for better management of the treatment and diagnosis.

\section{Discussion of the Research Study Questions}

In this section we discuss the research questions under study.

1. What are the variations in workflow among specialist and primary care offices in terms of workflow?

The variations among the primary care and specialist offices can be identified along two dimensions. One dimension is the variability in patient population served and the second is the organizational structure of the offices themselves. In general, primary care physician offices encounter higher patient variety and often do not have many in-house services for sample collection, X-ray or other services. On the other hand, specialty offices tend to include other services that they see as integral to providing care, for example, sample collection, X-ray, or 
chemotherapy. The variations in the tasks and task flow in specialty service tend to be low compared to a PCP or an internist, due to the prescreening by the PCP and the more standardized set of transactions. Furthermore, primary care offices seem to have more external interactions than the specialist offices.

UML diagrams are insufficient to provide a comparison between primary care and specialty offices, as they do not reflect the number of entities of a particular type that the physicians interact with. The interactions that a primary care office participates in are much richer and varied in contrast to the specialty offices. Most often specialty offices are connected to a hospital, where they have access to hospital information systems.

\section{What are the sources of delay (most time consuming tasks) that are part of the workflow?}

In Table 2, we presented the mode of operation for each task. Clearly all paper-based work requires considerable time to collate and file the various types of information. Due to the interleaved nature of many tasks, there is considerable scope for error, resulting in possible rework later. In general, Figure 2 provides an overview of the tasks that are most time consuming. There are multiple reasons for this, but the primary problem is the lack of adequate computer-based interfaces with external entities. Additionally, the external entities may not have a proper information infrastructure resulting in the need for using the telephone, fax and other manual means of getting information, leading to a considerable waste of time. This is most evident from the lack of computer-based insurance verification that appears first on the list. The other time-consuming tasks include communicating with laboratories and release of prior patient records from other physicians, both requiring telephone calls and unpredictable and time consuming request-to-receipt cycles.

Further time-consuming interactions include patient scheduling and follow-up of appointments, gathering prior patient history, referrals and other external interactions that take place through faxes, ground mail and sometimes e-mail. Further, these interactions necessitate the use of paperbased systems augmenting the EHR system, even when one is implemented.

One major hurdle for computerization is the lack of understanding of the IT issues involved. Further, most external interactions are not clearly defined and there is a lack of standard interfaces with external entities. This results in paper-based and manual interactions which are impediments for computerization. The cost of maintaining information in multiple forms and in multiple repositories leads to additional time consuming effort in organizing and charting tasks.

\section{What are the reasons for the extent of computerization or lack thereof in specialist and primary care offices?}

Most of the previous studies have shown low adoption rates in small offices without much differentiation between primary care and specialty offices. The case studies conducted in this paper show that many of the specialty offices are more advanced with respect to use of computerization than the primary care offices. The main reason identified is that most of the specialty care offices have either first introduced scheduling software or have introduced EHR systems with varying levels of augmentation with paper-based systems, with the exception of the 
ENT office which relies on the EHR system for organization and retrieval of information on patient charts.

The answer to the lack of computerization in primary care offices may be attributed to the complexity of the transactions due to the variety in patient populations and lack of standardized transactions across external agencies (insurance, hospitals and other specialists). Given the complexity of the transactions, most offices often tend to adopt minimal and fragmented computerization for tasks such as billing separated from medical records. With an increase in entities that a physician's office interacts, the problem of interoperability and the level of computerization of interacting entities come into play in estimating the usefulness and effectiveness of the EHR system. It was found in a time-and-motion study that the overall median time saved by the physician was 0.5 minutes, a decrease from 27.55 minutes to 27.05 minutes, with an EHR implementation [20], which may be due to inadequate interfaces with external entities. Until a tipping point is reached with respect to economics of a given office, EHR adoption will be slow. This is borne out by a study that claims that EHR implementation is generally viewed from a financial dimension and not from the quality of care point of view by the physicians [11]. A cause of the low rate of adoption is clearly the lack of network effects that reinforce the perception and reality of high capital investment and low potential returns in implementing EHR systems.

There are considerable benefits in implementing EHR systems in a physician's office, such as: increased overall operational efficiency, reduced medical error rate (which will significantly contribute to national healthcare savings), ability to generate templates and reminders, decreased staff utilization, less space consumption with the elimination of paper charts and easier production of “audit trails” for insurance coding. The EHR systems' financial costs and benefits can affect the rate at which health care providers adopt them, while quality improvement benefits can affect patients' health, which may in turn result in financial benefit to payers from avoiding “downstream” expenditures, especially for hospital and emergency room services [13]. In summary, the benefits of EHR implementation clearly outweigh any drawbacks encountered by physicians trying to implement EHR systems.

\section{Discussion and Conclusion}

There are several conclusions drawn from this study on workflow and EHR systems in small medical practices. First, the physician's offices with EHR implementations, mainly specialty offices, were fully satisfied with the process and claimed to have improved their efficiency. However, they felt that an upgrade in appropriate interfaces with external entities would lead to further time savings. For primary care offices, the major sources of work delay came from interactions with external entities, including previous care entities of the patient, multiple insurance companies, laboratories, hospitals, and outside referrals. Currently, none of these tasks can be done easily through the computer. Hence, paper-based systems will be the norm for these tasks in primary care settings. A specialty office operates through referrals from PCPs. The PCP office would clear the patient for initial insurance claims and send all the relevant records with the referral, hence reducing the task of chasing prior records and other information, as is the case in PCP offices,. Thereby, the time-consuming external interactions are reduced in the specialty 
offices. Mapping and correlation of patient identifiers and standards-based information interchange facilities can enable a large healthcare enterprise to provide a virtual patient record that overlies the fragmented records of the patient in the different healthcare facilities [1].

From an economic point of view, a primary care physician's cost of computerization is directly related to the ability to exploit network effects while interacting with external entities. The number of external entities that the physician's office has to interact with, and their level of computerization, is crucial in choosing the appropriate EHR system. The lack of computerization and inadequate interfaces of external participants reduces the economic benefits as the transaction cost for dealing with the external entities outweighs the benefits that could be accrued with computerization within the office. Also, there is a fear about the initial delay and loss of patients in the months after EHR implementation, as the staff needs to be trained. Further, the old information has to be entered into the new system, and concerns about the security of the information need to be addressed. Hence, the physicians will need to be prepared for the extra financial burden associated with EHR implementation [9].

The Nationwide Health Information Network (NHIN) program currently being designed and experimented by Department of Health and Human Services (DHHS) is an important activity that could increase adoption. The goal of the program is to aid the use and development of standards in determining the interoperability of the healthcare system. Without the network infrastructure available to link the different healthcare entities, adoption rates will remain relatively low in spite of possible advantages of EHR systems for the internal operation of offices. The linkages of the specialty offices to hospitals with laboratory information system or other computerized systems allowed them to schedule and order laboratory tests and also to receive reports electronically. This encouraged them to use at least a simple EHR system, which also helped them to integrate internal information. On the other hand, primary care offices with numerous interactions with a variety of entities are caught in the clutches of not being able to rid themselves of paper based systems leading to reluctance in adopting EHR systems.

There are some strategies that small physician offices can use to implement EHR systems:

Start small and grow: There are several low-cost EHR systems in the market which can be installed with considerable ease. One of the offices, totally paper-based, installed a low cost EHR system. However, this EHR system is only being used by the front office for scheduling and appointments, as the physician is reluctant to spend time on entering patient interactions due to time constraints. A possible solution is to use a voice recognition system specifically developed for the medical field. Once the physician is comfortable with using the EHR system, then appropriate linkages can be made to external entities such as billing software, a major requisite for small practices where billing is generally outsourced.

Look for certified EHR systems: In the past year, there has been a push toward using EHR systems that are certified by the Certification Council for Healthcare Information Technology (CCHIT) (http://www.cchit.org).

Participate in government funded programs: Considerable emphasis is being placed by the Federal government on using health information technology. For example, the Center for 
Medicare and Medicaid Services (CMS) (http://www.cms.hhs.gov) has announced a demonstration initiative where selected physician offices will be provided with monetary incentives for implementing EHR systems and confirming to CMS' quality metrics. The results of this five-year demonstration will provide useful data on EHR adoption.

\section{Disclaimer}

Certain commercial software systems are identified in this paper. Such identification does not imply recommendation or endorsement by the NIST; nor does it imply that the products identified are necessarily the best available for the purpose. Further, any opinions, findings, conclusions or recommendations expressed in this material are those of the authors and do not necessarily reflect the views of NIST or any other supporting US government or corporate organizations. 


\section{Appendix A: Questionnaire}

1. Which tasks do you perform in relation to healthcare delivery to the patient? Give a brief listing.

2. For each of the tasks, which documents or other information do you need/use?

3. For each of the above, where/who do you get this information from (e.g. patients, relatives, friends, physicians, hospitals, labs, etc.)?

4. How is the information transferred to you (e.g. collected, post, fax, email, oral/telephone, fileserver, etc.)?

5. How do you sort and/or store this information?

6. What kind of results do you produce? (e.g. different kinds of documents, referrals, prescriptions, etc.)

7. How are the results passed on to the next recipient? (e.g. automatically or on request)

8. Who checks the results/what kind of feedback do you get?

9. Which tools do you use for your tasks? (Computer-assisted and/or manual ones)

10. Which of your tasks seem most time consuming or inefficient?

11. Wishes (tools/new functionalities etc.) or other comments.

\section{Appendix B: Questions for the in situ interview}

1. What do you do when a new patient comes in?

2. What do you do when an old patient comes in?

3. How do you schedule an appointment?

4. How do you inform the patient the changes in the appointment?

5. How do you convey the test results to the patient or any matters of urgency?

6. How does the patient intimate the doctor's office, the test results?

7. How does the patient change or cancel appointments, how do you reschedule, and what do you do to update your records?

8. How are the appointment charts maintained?

9. What are the procedures you perform when a patient arrives, e.g., take out the chart, put in encounters, fill in insurance details, etc.?

10. What are all the encounters you use at each visit by a patient?

11. How do you attend telephone calls, and what do you do when you need to take details from the telephone calls?

12. How do you put reminders about each patient's annual checkups, annual tests, or followups?

13. Do you intimate the patient, remind about an upcoming event, and ask him/her to schedule an appointment for the same?

14. How are you informed each time the patient is hospitalized and about the condition of the patient?

15. How do you update hospitalization information in the charts?

16. How do you update medication and how do you call for medications after there has been a change to the previous medication after hospitalization?

17. How do you collect reports from the labs? Email, telephone, or fax?

18. How do you order investigations? Complete procedure about the concerned form being filled, scheduling the test, etc.? 
19. What are the functions of the RN? What encounter forms does he/she use?

20. How are the essential findings highlighted for the physician to notice it?

21. How does the physician record the findings?

22. How does the physician put the reminders, important events, or the findings to be noticed at the next visit?

23. How does the physician communicate with the hospital or the consulting physicians about the condition or any clarifications?

24. Does the physician follow-up on the condition of the patient after referring the patient to another specialist or hospital? If so, how?

25. Does the physician fill out the charts, in front of the patient?

26. How does the physician refer to the medication list of the patient before prescribing? How is the medication list updated and by whom?

27. How does the physician order medication?

28. How does the physician keep account of the medication previously ordered and when, so that the medications are not abused?

29. How does the physician correspond with the caregivers of the patient?

30. How and when does the physician fill in the encounters for the billing?

31. What is the task performed by the office manager's for billing?

32. How is the communication maintained with the billing agent?

33. How long does it take for billing claims to be approved?

34. What do you do to clarify billing discrepancies?

35. Who verifies the billing?

36. What is the view of the physician about EHR implementation? 


\section{References}

1. Bock, C., Carnahan, L., Fenves, S., Gruninger, M., Kashyap, V., Lide, B., Nell, J., Raman, R. and Sriram, R.D. "Healthcare Strategic Focus Area: Clinical Informatics," NISTIR 7263, National Institute for Standards and Technology, Gaithersburg MD, September 2005.

2. Hillestad R., Bigelow J., Bower A., Girosi F., Meili R., Scoville R., and Taylor R. "Can Electronic Medical Record Systems Transform Health Care? Potential Health Benefits, Savings, and Costs,” Health Affairs,_24(5), pp. 1103-1117, September 2005.

3. Hing, E. and Burt, C.W. "Office-based Medical Practices: Methods and Estimates from the National Ambulatory Medical Care Survey,” Advanced Data from Vital and Health Statistics, Number 383, CDC, Department of Health and Human Services, March, 2007.

4. Burt, C. W., and Sisk, J.E. "Which Physicians And Practices Are Using Electronic Medical Records?” Health Affairs, 24 (5), pp. 1334-1343. September 2005.

5. Lee J.N., Cain C., Young S., Chockley N. and Burstin H. "The Adoption Gap: Health Information Technology in Small Physician Practices,” Health Affairs, 24 (5), pp. 13641366, September 2005.

6. Himmelstein, D.U., and Woolhandler, S. "Hope and Hype: Predicting The Impact of Electronic Medical Records,” Health Affairs,_24(5), pp. 1121 - 1123, September 2005.

7. Wang, S.J., et. al., "Cost Benefit Analysis of Electronic Medical Records in Primary Care,” The American Journal of Medicine, 14, pp 397-403, April 2003.

8. Bates, D. W. "Physician and Ambulatory Electronic Health Record," Health Affairs,_24 (5), pp. 1180-1189, September 2005.

9. Loomis, G.A., Ries, S., Saywell. Jr. R.M. and Thakkar, N.R. "If electronic medical records are so great, why aren't family physicians using them?” Journal of Family Practice, 51(7), July 2002.

10. Morrissey, J. "A Day in the Life of a Medical Record," http://www.nahit.org/cms/index.php?option=com_content\&task=view\&id=258\&Itemid= 211, The National Alliance for Health Information Technology, 2006.

11. Sidorov, J. "It Ain't Necessarily So: The Electronic Health Record And The Unlikely Prospect Of Reducing Health Care Costs,” Health Affairs, 25(4), pp. 1079 - 1085, July 2006.

12. Miller, R. and Sim, I. "Physicians' use of Electronic Records: Barriers and Solutions," Health Affairs, 23(2), pp 116-126, 2004.

13. Miller, R. H., West, C., Brown, T. M., Sim, I. and Ganchoff, C. “The Value of Electronic Health Records in Solo or Small Group Practices.” Health Affairs,_24(5), pp. 1127 1137, September 2005.

14. Pizziferri, L., Kittler, A. F., Volk, L. A., Honour, M. M., Gupta, S.R., Wang, S., Wang, T., Lippincott, M., Qi, L. and Bates, D. W. "Primary care physician time utilization before and after implementation of an electronic health record: A time-motion study." Journal of Biomed Inform. 38(3):176-88 June 2005.

15. Poon, E.G., Jha, A. K., Christino, M., Honour, M., Fernandopulle, R., Middleton, B., Newhouse, J., Leape, L., Bates, D.W., Blumenthal, D. and Kaushal, R. "Assessing the level of healthcare information technology adoption in the United States." BMC Med Inform Decis Mak, 6(1), Jan. 5, 2006. 
16. Valdes, I., Kibbe, D.C., Tolleson, G., Kunik, M.E. and Petersen, L.A. "Barriers to Proliferation of Electronic Medical Records,” Informatics in Primary Care, 12(1), pp. 39(7), February 2004.

17. Simon, S. R., Kaushal, R., Cleary, P. D., Jenter, C. A., Volk, L. A., Poon, E. G., Orav, E. J., Lo, H. G.,. Williams, D. H. and Bates, D.W. "Correlates of Electronic Health Record Adoption in Office Practices: A Statewide Survey,” J. Am. Med. Inform. Assoc., 14(1), pp. 110 - 117, January 2007.

18. Yin, R. K. “Case Study Method: design and methods,” Sage publications, Third edition, 2003.

19. Booch, G., Rumbagh, J., and Iverson, J. “The Unified Modeling Language User Guide,” Addison-Wesley Object Technology Series, 1998.

20. Lo, H. G., Newmark, L. P., Yoon, C., Volk, L., Carlson, V. L., Kittler, A. F., Lippincott, M., Wang, T. and Bates, D.W. "Electronic Health Records in Specialty Care: A TimeMotion Study”, Journal of American Medical Informatics Association, Vol 14, pp 609615, June 2007. 


\begin{tabular}{|c|c|c|c|c|c|c|c|c|c|c|c|c|c|}
\hline $\begin{array}{l}\text { Organizatio } \\
\text { n }\end{array}$ & $\begin{array}{l}\text { Pr. } \\
\text { Care* }\end{array}$ & Pr. C & FP-1 & FP-2 & Neuro & Multisp & OB/GYN & Gastro & Cardio & Heme/Onc & $\begin{array}{l}\text { Ophth } \\
\text { al }\end{array}$ & Nephro & ENT \\
\hline Physicians & 2 & 1 & 2 & 1 & 2 & 10 & 1 & 1 & 2 & 2 & 1 & 6 & 1 \\
\hline Front Desk/ & 2 & 2 & 2 & 2 & 2 & 2 & $1+1$ & 2 & 3 & 2 & 2 & 3 & 2 \\
\hline Nurse & 1 & 1 & 1 & 1 & 1 & 10 & $\begin{array}{l}1 \\
1 \\
\text { billing/off } \\
\text { ice }\end{array}$ & 1 & 1 & 3 & 1 & 2 & 1 \\
\hline Billing Mgr & 0 & 0 & 1 & 0 & 0 & 2 & manager & 0 & 1 & 0 & 0 & 3 & \\
\hline Other & 0 & 0 & 0 & 0 & $\begin{array}{l}\text { 1-office } \\
\text { manager }\end{array}$ & $\begin{array}{l}2 \text {-record } \\
\text { keepers }\end{array}$ & 0 & 1 & $\begin{array}{l}\text { 1-physical } \\
\text { therapist }\end{array}$ & 0 & 0 & 1 & $\begin{array}{l}\text { Audiologi } \\
\text { st } \\
\text { Audio- }\end{array}$ \\
\hline $\begin{array}{l}\text { Lab/other } \\
\text { facilities }\end{array}$ & 0 & 0 & 0 & 0 & $\begin{array}{l}\text { EMG/EEG } \\
\text { Facility }\end{array}$ & $\begin{array}{l}\text { Sample } \\
\text { collection, } \\
\text { X-ray, \& } \\
\text { pharmacy }\end{array}$ & $\begin{array}{l}\text { Sample } \\
\text { Collection } \\
\text { \&simple } \\
\text { tests }\end{array}$ & $\begin{array}{l}\text { Endoscopy/ } \\
\text { Colonoscopy }\end{array}$ & $\begin{array}{l}\text { ECO/ } \\
\text { Physical } \\
\text { therapy/Stress } \\
\text { Test }\end{array}$ & $\begin{array}{l}\text { Sample } \\
\text { Collection } \\
\text { \&Chemo- } \\
\text { therapy }\end{array}$ & $\begin{array}{l}\text { Vision } \\
\text { testing }\end{array}$ & 0 & $\begin{array}{l}\text { metry/ } \\
\text { ultrasound } \\
\text { /minimal } \\
\text { procedures }\end{array}$ \\
\hline
\end{tabular}

Table 1: Physician Office Compositions/ Facilities 


\begin{tabular}{|c|c|c|c|c|c|c|c|c|c|c|c|c|c|}
\hline TASKS & Primary Care -1 & $\begin{array}{l}\text { Primary } \\
\text { Care-2 }\end{array}$ & $\begin{array}{l}\text { Family } \\
\text { Practice-1 }\end{array}$ & $\begin{array}{l}\text { Family } \\
\text { Practice-2 }\end{array}$ & Neuro & Multisp & OB/GYN & Gastro & Cardio & Heme/Onc & Ophthal & Nephro & ENT \\
\hline Registration & 1 & 2 & 1 & 1 & 2 & 2 & 2 & 2 & 2 & 2 & 2 & 2 & 2 \\
\hline Ins Verification & 0 & 0 & 0 & 0 & 0 & 0 & 0 & 0 & 0 & $1 / 0$ & $1 / 0$ & 0 & 0 \\
\hline Vitals & 1 & 1 & 1 & 1 & 1 & 1 & 1 & 1 & 1 & 1 & 1 & 1 & 2 \\
\hline History & 1 & 1 & 1 & 1 & 1 & 1 & 1 & 1 & 1 & 1 & 1 & 1 & $3 / 2$ \\
\hline Consultation & 1 & 1 & 1 & 1 & $1 / 3$ & 1 & 1 & 1 & 1 & 2 & 2 & 2 & 2 \\
\hline Prescription & 1 & 1 & 1 & 1 & 1 & 1 & 1 & 1 & 1 & 1 & 1 & 1 & 1 \\
\hline Procedure & 1 & 1 & 1 & 1 & 1 & 1 & 1 & 2 & 2 & 1 & 2 & 2 & 2 \\
\hline Referral & $0 / 1$ & $0 / 1$ & $0 / 1$ & $0 / 1$ & 0/1 & $0 / 1$ & $0 / 1$ & $0 / 1$ & $0 / 1$ & $0 / 1$ & $0 / 1$ & $0 / 1$ & $0 / 1$ \\
\hline Certificates & 1 & 2 & 1 & 1 & 1 & 1 & 1 & 2 & 1 & 2 & 2 & 2 & 2 \\
\hline Appointments & 1 & 2 & 1 & 1 & 2 & 1 & 2 & 2 & 2 & 2 & 2 & 2 & 2 \\
\hline Labs & 1 & 1 & 1 & 1 & 1 & 2 & 1 & 1 & 1 & 1 & 1 & 1 & 1 \\
\hline Billing & $4 / 1$ & $4 / 2$ & 1 & 1 & $4 / 3 / 2$ & 2 & 2 & $4 / 1$ & 2 & 2 & 2 & 2 & $3 / 2$ \\
\hline
\end{tabular}

Legend: 0: Telephone, 1: Paperwork, 2 : Computer support, 3: Transcription, 4: External Billing

Table 2: Small office physician tasks and mode of work 